\title{
The clinical features and prognosis of type 4C myocardial infarction in patients with non-ST-segment elevation myocardial infarction
}

\author{
Jixiang Wang ${ }^{1,2}$, Honggang $\mathrm{Gao}^{3}$, Jianyong $\mathrm{Xiao}^{2}$, Mingdong $\mathrm{Gao}^{2}$, Yin $\mathrm{Liu}^{2,4}$, Jing Gao ${ }^{4,5,6}$ \\ ${ }^{1}$ Tianjin Medical University, Tianjin, China; ${ }^{2}$ Department of Cardiology, Tianjin Chest Hospital, Tianjin, China; ${ }^{3}$ Department of Cardiology, Qufu \\ People's Hospital, Qufu, China; ${ }^{4}$ Thoracic Clinical College, Tianjin Medical University, Tianjin, China; ${ }^{5}$ Chest Hospital, Tianjin University, Tianjin, \\ China; ${ }^{6}$ Cardiovascular Institute, Tianjin Chest Hospital, Tianjin, China \\ Contributions: (I) Conception and design: J Wang; (II) Administrative support: Y Liu; (III) Provision of study materials or patients: J Gao; (IV) \\ Collection and assembly of data: H Gao; (V) Data analysis and interpretation: J Xiao; (VI) Manuscript writing: All authors; (VII) Final approval of \\ manuscript: All authors. \\ Correspondence to: Prof. Jing Gao, PhD, MD. Cardiovascular Institute, Tianjin Chest Hospital, No. 261 Taierzhuang Road, Jinnan District, Tianjin \\ 300222, China. Email: gaojing2088@163.com; Prof. Yin Liu, MD. Department of Cardiology, Tianjin Chest Hospital, No. 261 Taierzhuang Road, \\ Jinnan District, Tianjin 300222, China. Email: liuyin2088@163.com.
}

Background: Type 4C myocardial infarction (MI) is a special type of myocardial infarction related to restenosis without thrombosis. There is a lack of relevant data on this new classification of acute MI (AMI). This study set out to examine the prognosis and treatment of type 4C MI in patients with non-ST-segment elevation MI (NSTEMI).

Methods: With reference to the NSTEMI cohort study database, we enrolled 1,032 cases of type $1 \mathrm{MI}$ and 42 cases of type 4C MI from the period January 01, 2018 to August 31, 2018. All cases were followed up for 1 year. The outcome was major cardiovascular adverse events (including all-cause deaths, nonfatal MI, heart failure necessitating hospitalization, uncontrollable angina pectoris, and revascularization of the target vessels). Risk ratios (RR) were calculated using the generalized linear model. Cox multivariate analysis was performed to analyze the prognostic effects of drug-coated balloon (DCB) angioplasty or drug-eluting stent (DES) implantation in patients with type 4C MI.

Results: Compared with type $1 \mathrm{MI}$, type 4C MI was associated with a higher incidence of major adverse cardiovascular events (MACEs) [21.43\% vs. 5.14\%; adjusted RR: 3.725, 95\% confidence interval (CI): $1.937-$ 7.164]. Type 4C MI also showed a higher 1-year mortality rate than type $1 \mathrm{MI}$ (7.14\% vs. $1.55 \%$; unadjusted RR: 4.607, 95\% CI: 1.395-15.212). However, after adjusting for covariates, no statistical difference was noted (adjusted RR: 2.515, 95\% CI: 0.768-8.233). Multiple adjustments to the Cox multivariate models revealed that neither DCB nor DES affected the clinical outcomes.

Conclusions: Type 4C MI has a poorer prognosis than type 1 MI. DCB angioplasty and DES implantation show similar efficacy in the treatment of type 4C MI.

Keywords: Type 4C myocardial infarction (type 4C MI); universal definition of myocardial infarction; restenosis

Submitted May 07, 2021. Accepted for publication Jun 21, 2021.

doi: $10.21037 / \mathrm{atm}-21-2587$

View this article at: https://dx.doi.org/10.21037/atm-21-2587 


\section{Introduction}

Acute myocardial infarction (AMI) is rising in incidence, which has increased the global burden of disease, and the demand for prevention and treatment services is continuing to grow (1). The definition and classification of AMI aid doctors in the medical process, and the condition can usually be classified into 5 types based on its pathogenesis (2). The Fourth Universal Definition of Myocardial Infarction, published in 2018, proposed type 4C myocardial infarction (MI), which is related to restenosis without other criminal lesions or thrombotic causes on the basis of the original classification (3). The Fourth Universal Definition of Myocardial Infarction can not only guide individualized treatment of patients with the disease, but it also provides ideas for interdisciplinary diagnosis and treatment (4). Diagnosing type 4C MI is challenging, and no research report has characterized its population characteristics, morbidity, or prognosis (5). Restenosis AMI usually presents as non-ST-segment elevation MI (NSTEMI) $(6,7)$. Stent stenosis triples the mortality rate of patients with NSTEMI. However, stent restenosis without MI is not associated with an increased risk of death (8). The clinical prognosis of AMI caused by in-stent restenosis (ISR) has also been reported to be worse than that of angina pectoris, with a 4-fold increase in the incidence of major adverse cardiovascular events (MACEs) at 6 months after intervention (9). Therefore, type 4C MI may have a poor prognosis, but there is a lack of relevant data on this new classification of AMI. Further, no study so far has assessed whether the outcomes of nextgeneration drug-eluting stent (DES) implantation and drugcoated balloon (DCB) angioplasty differ in patients with type 4C MI.

The clinical characteristics and prognosis of type 4C MI, as a non-thrombotic, restenosis-related MI, remain unclear. In this first-of-its-kind study, we aimed to elucidate the clinical characteristics and prognosis of type 4C MI by comparing type $4 \mathrm{C}$ and type $1 \mathrm{MI}$, and comparing the therapeutic effects of DCB angioplasty and DES implantation in patients with the condition. We present the following article in accordance with the STROBE reporting checklist (available at https:// dx.doi.org/10.21037/atm-21-2587).

\section{Methods}

\section{Data sources and participants}

The data in this study were obtained from the database of “Acute Myocardial Infarction in Tianjin" (TAMI), which is a multicenter, real-world cohort study (Clinical Trials Registration: NCT03600259). Data from continuously included patients with AMI from January 1, 2018 to August 31, 2018 were collected for our analysis. Data included patients' baseline characteristics, treatment, and follow-up information.

All cases were reclassified by 2 senior cardiologists (J.X. and M.G.) with reference to the Fourth Universal Definition of Myocardial Infarction. In cases of dispute between the 2 experts, the diagnosis was classified through discussion with a third cardiologist (Figures S1-S3). In the initial review, no cases of type 4C MI were found among patients with STEMI; thus, type 1 and type 4C MI were compared in patients with NSTEMI.

The following inclusion criteria were used to select participants for the study: (I) patients meeting the diagnostic criteria for type 1 or $4 \mathrm{C} \mathrm{MI}$, as per the Fourth Universal Definition of Myocardial Infarction (3); and (II) age $>18$ years. The exclusion criteria were as follows: (I) patients who did not undergo coronary angiography; (II) unwillingness to cooperate with the treatment regime; and (III) pregnant or lactating women. Signed informed consent was obtained from all participants. The study protocol met the requirements of and was approved by the Tianjin Chest Hospital Ethics Committee (approval No. 2018KY-010-01). All procedures performed in this study involving human participants were in accordance with the Declaration of Helsinki (as revised in 2013).

\section{Treatment}

All patients received the best possible medication, invasive examination, and treatment based on the relevant guidelines (10). Invasive examinations and treatment were delivered by experienced high-caliber physicians working in cooperation with professional assistants, in strict accordance with the operating procedures.

The patients were routinely prescribed $300 \mathrm{mg}$ aspirin and $300 \mathrm{mg}$ clopidogrel sulfate (or $180 \mathrm{mg}$ ticagrelor) for antiplatelet aggregation. During the operation, heparin calcium was used for anticoagulation, and the activated clotting time (ACT) was measured at $>300$ s. Coronary angiography was carried out to determine the target lesion and the degree of vascular stenosis. The balloon was inserted along the guidewire, and the DES was inserted after pre-dilation at the lesion site; post-dilation of the balloon was selected based on the dilation of the stent. For lesions with ISR, a second-generation DES was implanted 
or DCB dilatation was applied. The major reason for using a DCB was that the target lesion was confined to the original stent and did not involve the coronary artery outside the stent.

\section{Clinical outcomes and follow-up}

Commencing from the day of hospitalization, the patients were followed up for 1 year via telephone, home visits, outpatient reviews, and other relevant procedures. The main clinical outcome was MACEs, including all-cause death, non-fatal MI, heart failure requiring hospitalization, angina beyond drug control, and revascularization of the target vessels. In all cases, MI was diagnosed with reference to the Fourth Universal Definition of Myocardial Infarction (3). Heart failure was diagnosed with reference to European Heart Association's guidelines for the diagnosis and treatment of heart failure (11). Uncontrollable angina was defined as the onset of angina after receipt of the maximum tolerable dose of beta blockers and nitrates. Target lesion revascularization referred to revascularization (percutaneous coronary intervention or coronary surgery) performed for recurrent stenosis of the target lesion segments (12).

\section{Statistical analyses}

SPSS 25.0 software (IBM Corporation) was employed for data analysis. The 2 observation cohorts were the type $1 \mathrm{MI}$ group and the type 4C MI group. Under the condition of normal distribution, measurement data were expressed as the mean \pm standard deviation (SD) and the $t$ test was used; otherwise, they were expressed as median (interquartile range), and the Wilcoxon signed-rank test was used. Enumeration data were presented as percentages (\%), and the Chi-square test or Fisher's exact test was adopted for comparisons between the 2 groups.

The incidence of MACEs was calculated, and KaplanMeier survival curves were drawn to analyze the prognosis of patients with type 4C MI. The risk ratios (RR) for the incidences of clinical events were calculated using the generalized linear model, adjusted for the Global Registry of Acute Coronary Events (GRACE) Score (which was calculated based on age, creatinine levels, heart rate, systolic blood pressure, Killip grade, pre-hospital cardiac arrest, elevation of myocardial injury markers, and electrocardiogram ST segment changes), diabetes, history of MI, history of stroke, left ventricular ejection fraction (LVEF), and N-terminal pro-brain natriuretic peptide (NT-
proBNP) levels . The Cox proportional hazard regression model was employed to analyze the relationship between the vascular interventional therapy strategy (DCB or DES) and MACE events in the type 4C MI cohort. Receiver operating characteristic (ROC) curve analyses were conducted to test the predictive ability of prognostic factors, with their predictive power indicated by the area under the ROC curve (AUC). $\mathrm{P}<0.05$ indicated statistical significance.

\section{Results}

\section{Baseline characteristics}

A total of 1,074 patients were included in this study, including 42 patients with type $4 \mathrm{C}$ MI and 1,032 patients with type $1 \mathrm{MI}$. The rate of type $4 \mathrm{C} \mathrm{MI}$ in patients with AMI was about $1.02 \%$ (Figure S1). Comparison of the baseline data between the 2 groups revealed that the incidence of stroke and history of $\mathrm{MI}$ and percutaneous coronary intervention were higher in the type 4C MI group than the type $1 \mathrm{MI}$ group. In both groups, the LVEF value was lower than that in type $1 \mathrm{MI}$ group $(\mathrm{P}<0.05)$. Other variables including age and sex showed no statistically significant difference between the 2 groups (Table 1).

\section{Clinical outcomes}

After 1 year of follow-up, the incidence of MACEs was significantly higher in the type 4C MI group than in the type $1 \mathrm{MI}$ group [21.43\% vs. 5.14\%, adjusted RR: 3.725 , 95\% confidence interval (CI): 1.937-7.164], whereas allcause mortality was higher in the former group, albeit there was no statistical difference after correction $(7.14 \%$ vs. $1.55 \%$, adjusted RR: 2.515 , 95\% CI: 0.768-8.233). No statistical difference was noted in the incidence of uncontrollable angina pectoris or non-fatal MI after correction. Heart failure and target lesion revascularization were higher in incidence in patients with type 4C MI than in patients with type $1 \mathrm{MI}$, with the difference being statistically significant (Table 2). Kaplan-Meier survival curve analysis revealed significant differences in MACEs and all-cause deaths between the 2 groups at the beginning of the follow-up period ( $\mathrm{P}<0.05$; Figure 1).

\section{Comparison of treatment methods}

DES and DCB were associated with similar MACE rates in the type $4 \mathrm{C}$ MI cohort ( $16.7 \%$ vs. $28.6 \%, \mathrm{P}=0.433)$. 
Table 1 Comparison of the baseline data of the study participants

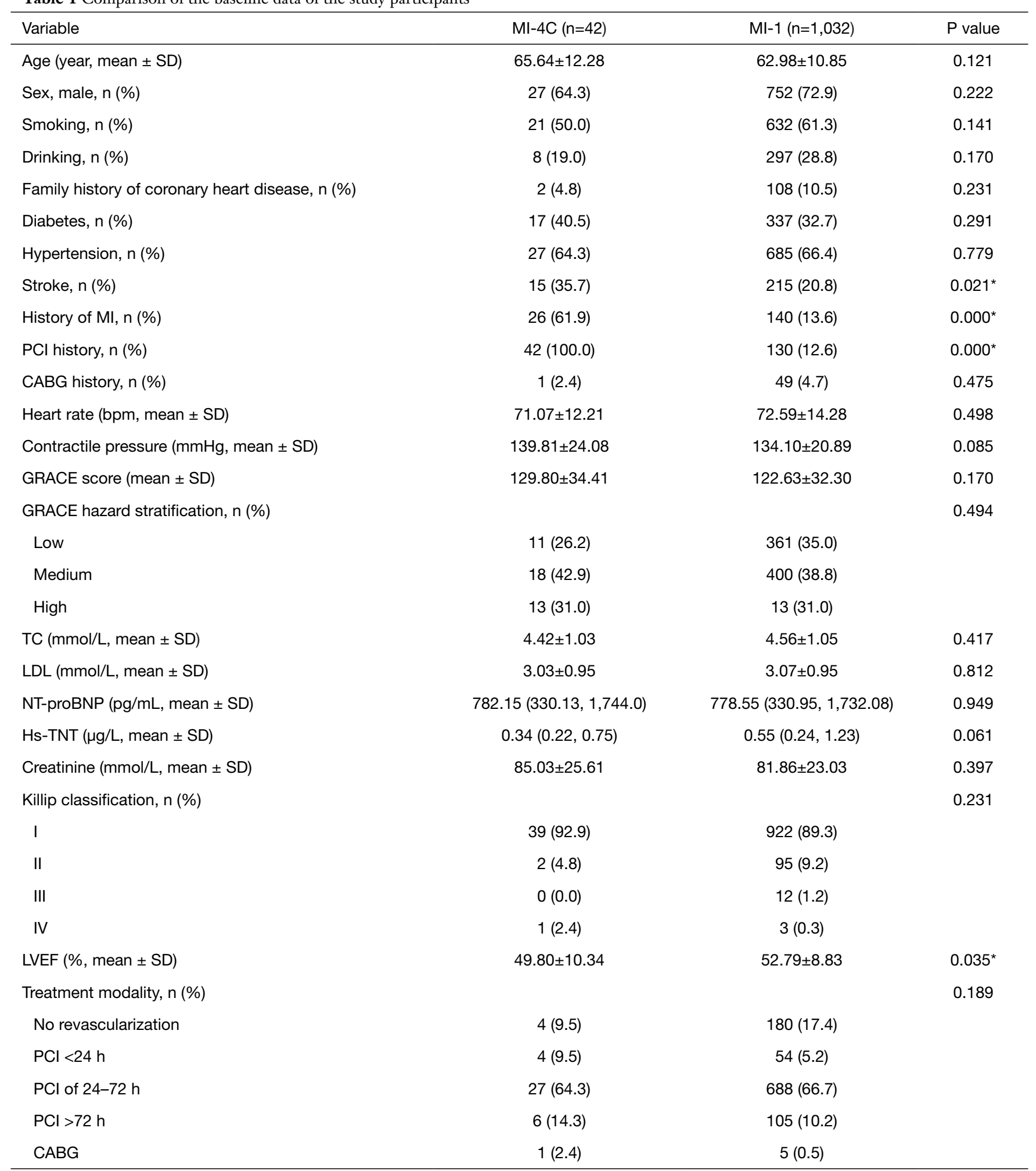

${ }^{*} \mathrm{P}<0.05$. MI-4C, type 4C myocardial infarction; MI-1, type 1 myocardial infarction; MI, myocardial infarction; TC, total cholesterol; LDL, low-density lipoprotein; NT-proBNP, N-terminal pro-brain natriuretic peptide; hs-TNT, hypersensitive troponin T; LVEF, left ventricular ejection fraction; $\mathrm{PCl}$, percutaneous coronary intervention; CABG, coronary artery bypass grafting. 
Table 2 Comparison of the clinical outcomes between the 2 groups

\begin{tabular}{lcccc}
\hline Variable & Ml-4C, $\mathrm{n}(\%)$ & $\mathrm{Ml}-1, \mathrm{n}(\%)$ & $\mathrm{RR}(95 \% \mathrm{Cl})$ & Correction RR (95\% Cl) \\
\hline MACE & $9(21.43)$ & $53(5.14)$ & $4.173(2.208-7.882)$ & $3.725(1.937-7.164)$ \\
All-cause death & $3(7.14)$ & $16(1.55)$ & $4.607(1.395-15.212)$ & $2.515(0.768-8.233)$ \\
Uncontrolled angina pectoris & $2(4.76)$ & $22(2.13)$ & $2.234(0.543-9.195)$ & $2.812(0.671-11.781)$ \\
Heart failure requiring hospitalization & $2(4.76)$ & $11(1.07)$ & $4.468(1.022-19.535)$ & $4.407(1.254-15.492)$ \\
Nonfatal myocardial infarction & $2(4.76)$ & $7(0.68)$ & $7.020(1.503-32.800)$ & $4.978(0.948-26.141)$ \\
Revascularization of target vessels & $1(2.38)$ & $1(0.10)$ & $24.571(1.561-386.624)$ & $28.768(3.858-214.521)$ \\
\hline
\end{tabular}

Correction factors: GRACE score, diabetes, history of myocardial infarction, stroke, left ventricular ejection fraction, N-terminal pro-brain natriuretic peptide; MI-4C, type 4C myocardial infarction; MI-1, type 1 myocardial infarction; MACE, major adverse cardiovascular event.

The Cox proportional risk review model revealed that the selection of DES or DCB did not influence the occurrence of MACEs and that an increased level of NT-proBNP was an independent risk factor for MACEs within 1 year (Table 3). As shown in Figure 2, NT-proBNP exhibited a good predictive performance (AUC: 0.779; 95\% CI: 0.573-0.986, $\mathrm{P}=0.016$ ).

\section{Discussion}

In a population of patients with NSTEMI who underwent coronary angiography, we re-classified type 4C MI cases with reference to the Fourth Universal Definition of Myocardial Infarction. We observed that the 1-year rates of MACEs and all-cause mortality were significantly higher with type 4C MI than with type $1 \mathrm{MI}$, with the former being more closely associated with target revascularization and heart failure. Moreover, the use of second-generation DES or DCB dilation treatment did not have any effect on the clinical prognosis, whereas increased NT-proBNP was found to act as a predictor of poor prognosis of type 4C MI.

To date, only a few studies have focused on the uniform definition of type 4C MI. A past retrospective study of 89 cases of AMI caused by ISR reported that the incidence and mortality of MACEs during the 6-month follow-up reached $21.6 \%$ and $13.8 \%$, respectively, which were far greater than the rates in the type 4C MI cohort in this study (9). Notably, patients with AMI due to thrombotic causes were not excluded from previous study; hence, cases in the cohort were not strictly type 4C MI, rather the results suggested a poor prognosis for ISR-related AMI.

Our study revealed that type $4 \mathrm{C} \mathrm{MI}$ is associated with a history of MI or stroke, and entails a low ejection fraction, which could further complicate the condition of patients with this type of MI, affecting their clinical outcomes . The application of a generalized linear model is required to correct for factors that may affect the prognosis. Further, we confirmed that type $4 \mathrm{C} \mathrm{MI}$ has a worse prognosis than type $1 \mathrm{MI}$, and carries a high risk of heart failure and target lesion revascularization events. It also has independent effects on adverse cardiac events, with MACEs occurring in at least $20 \%$ of patients within the 1 -year follow-up. Multivariate COX regression analysis found that elevated NT-proBNP is a risk factor for poor prognosis of type 4C MI. This may be related to more hospitalizations for heart failure after discharge.

Although there are several treatments available for ISR, the most effective strategy has yet to be established. Intracavitary imaging examination can help to clarify the pathogenesis of ISR and guide the treatment regimen (13-15). For cases of restenosis caused by stent underexpansion, poor adhesion, or stent rupture, balloon angioplasty or stent reimplantation is usually the treatment of choice. However, in most cases, ISR occurs due to severe neointimal hyperplasia, with a high percentage of recurrent target vessels being affected after balloon angioplasty alone $(16,17)$. At present, the best treatment option for ISR is the new generation of DESs or DCB dilatation. However, second-generation DES implantation may be superior to DCB dilatation $(18,19)$, with the advantages and disadvantages of these 2 treatment options potentially being related to the original stent type (20). Previous studies have mostly focused on patients with angina, but a few studies have applied DCBs in patients with AMI. The "DEB-AMI" study (21) demonstrated that DES implantation is superior to DCB angioplasty in patients with STEMI . However, a single-center retrospective study (22) reported that DES implantation and DCB angioplasty had comparable efficacy in patients with ISR-related AMI, with 

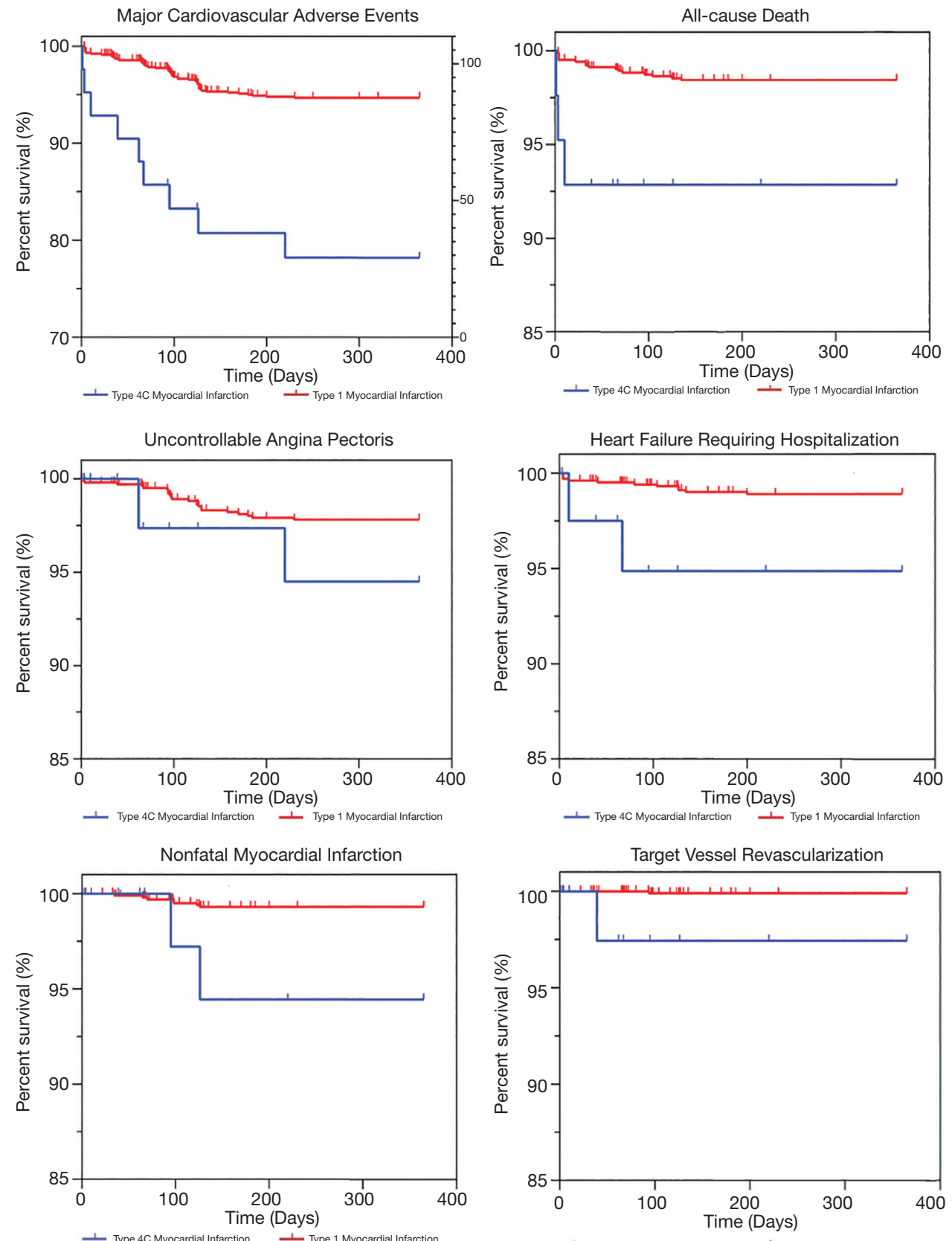

Figure 1 Major adverse cardiovascular events (MACEs) and Kaplan-Meier survival curve of all-cause mortality.

no statistical difference in the incidence of MACEs within 1 year $(37.5 \%$ vs. $27.5 \%, \mathrm{P}=0.474)$. In contrast with the results of these studies, the observed study population had ISR without thrombotic factors. After multiple adjustments to the correction model, no significant difference was noted in the therapeutic effects of the DES and DCB procedures. Despite the lack of randomized controlled trials on AMI, when combined with the current clinical evidence, our findings suggest that both DESs and DCBs are safe and reliable treatment options.

We have identified the following limitations to this study. First, the diagnosis of the study population were reclassified retrospectively. Although we set up an expert group to reclassify the types of MI, there is a probability 


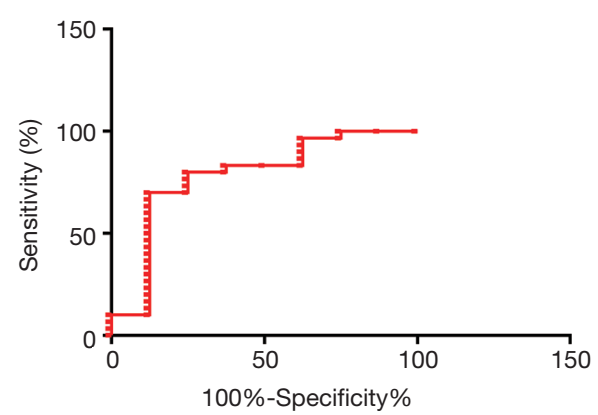

Figure 2 Receiver operating characteristic (ROC) curve analysis of $\mathrm{N}$-terminal pro-brain natriuretic peptide (NT-proBNP).

Table 3 Relationship between DES implantation/DCB angioplasty and 1-year MACEs in the type 4C myocardial infarction cohort

\begin{tabular}{|c|c|c|}
\hline Model & Variable and hazard ratio & $P$ value \\
\hline Model $^{1}$ & DCB HR: 1.802 (95\% Cl: 0.450-7.210) & 0.405 \\
\hline \multirow[t]{3}{*}{ Model $^{2}$} & DCB HR: 1.555 (95\% Cl: 0.370-6.544) & 0.547 \\
\hline & Age HR: 1.011 (95\% Cl: 0.945-1.083) & 0.745 \\
\hline & Male HR: 0.417 (95\% Cl: 0.084-2.071) & 0.285 \\
\hline \multirow[t]{4}{*}{ Model $^{3}$} & DCB HR: 1.550 (95\% Cl: 0.366-6.564) & 0.552 \\
\hline & Age HR: 1.011 (95\% Cl: 0.939-1.087) & 0.777 \\
\hline & Male HR: 0.417 (95\% Cl: 0.084-2.070) & 0.284 \\
\hline & GRACE HR: 1.0006 (95\% Cl: 0.981-1.021) & 0.951 \\
\hline \multirow[t]{8}{*}{ Model $^{4}$} & DCB HR: 3.615 (95\% Cl: 0.502-26.028) & 0.202 \\
\hline & Age HR: 0.983 (95\% Cl: 0.878-1.101) & 0.769 \\
\hline & Male HR: 0.348 (95\% Cl: 0.027-4.426) & 0.416 \\
\hline & GRACE HR: 0.998 (95\% Cl: 0.956-1.042) & 0.925 \\
\hline & Diabetes HR: 0.168 (95\% Cl: 0.011-2.542) & 0.198 \\
\hline & LVEF HR: 1.046 (95\% Cl: 0.921-1.188) & 0.487 \\
\hline & LDL HR: 1.319 (95\% Cl: 0.553-3.149) & 0.532 \\
\hline & $\begin{array}{l}\text { NT-proBNP HR: } 1.0004 \\
(95 \% \mathrm{Cl}: 1.0001-1.0008)\end{array}$ & $0.014^{*}$ \\
\hline
\end{tabular}

${ }^{\star} \mathrm{P}<0.05$. ${ }^{1}$, uncorrected for other factors; ${ }^{2}$, corrected for age and sex; ${ }^{3}$, corrected for age, sex, and GRACE score; ${ }^{4}$, corrected for age, sex, GRACE score, diabetes, LVEF, LDL, and NT-proBNP. DES, drug-eluting stent, DCB, drug-coated balloon; GRACE, Global Registry of Acute Coronary Events Score; LVEF, left ventricular ejection fraction; LDL, low-density lipoprotein; NT-proBNP, N-terminal pro-brain natriuretic peptide.

that population classification errors occurred. However, to avoid bias in the classification, the expert group was blinded to the treatment outcomes, follow-up, and other related details. Second, due to the low incidence of type 4C MI and the small number of included cases, there is a possibility of bias in the NSTEMI population. Thirdly, this study was not a randomized controlled study and it included a small sample size, which introduced the possibility of deviations in the evaluation of the effects of DCB angioplasty and DES implantation. To minimize interference from other factors, multiple corrections have be made for the variables that may affect the final outcome.

\section{Conclusions}

In summary, this study has shown that patients with type 4C MI have a poor prognosis and that the identification of high-risk patients with AMI may facilitate the development of preventive treatment regimens and improve prognosis. DCB angioplasty and DES implantation both appear to be effective treatment approaches for type 4C MI.

\section{Acknowledgments}

The authors thank all participants for their effort in the study. Funding: None.

\section{Footnote}

Reporting Checklist: The authors have completed the STROBE reporting checklist. Available at https://dx.doi. org/10.21037/atm-21-2587

Data Sharing Statement: Available at https://dx.doi. org/10.21037/atm-21-2587

Conflicts of Interest: All authors have completed the ICMJE uniform disclosure form (available at https://dx.doi. org/10.21037/atm-21-2587). The authors have no conflicts of interest to declare.

Ethical Statement: The authors are accountable for all aspects of the work in ensuring that questions related to the accuracy or integrity of any part of the work are appropriately investigated and resolved. Signed informed consent was obtained from all participants. The study protocol met the requirements of and was approved by the Tianjin Chest Hospital Ethics Committee (approval No. 2018KY-010-01). All procedures performed in this study involving human participants were in accordance with the Declaration of Helsinki (as revised in 2013). 
Open Access Statement: This is an Open Access article distributed in accordance with the Creative Commons Attribution-NonCommercial-NoDerivs 4.0 International License (CC BY-NC-ND 4.0), which permits the noncommercial replication and distribution of the article with the strict proviso that no changes or edits are made and the original work is properly cited (including links to both the formal publication through the relevant DOI and the license). See: https://creativecommons.org/licenses/by-nc-nd/4.0/.

\section{References}

1. Roth GA, Mensah GA, Johnson CO, et al. Global Burden of Cardiovascular Diseases and Risk Factors, 1990-2019: Update From the GBD 2019 Study. J Am Coll Cardiol 2020;76:2982-3021.

2. Yu Y, Wang J, Wang Q, et al. Admission oxygen saturation and all-cause in-hospital mortality in acute myocardial infarction patients: data from the MIMIC-III database. Ann Transl Med 2020;8:1371.

3. Thygesen K, Alpert JS, Jaffe AS, et al. Fourth Universal Definition of Myocardial Infarction (2018). Circulation 2018;138:e618-e651.

4. Eckner D, Pauschinger M, Ademaj F, et al. Clinical implications of the fourth universal definition of myocardial infarction. Herz 2020;45:520-7.

5. Bobrowska B, Rakowski T, Dziewierz A. A myocardial infarction in a patient with previous myocardial revascularization presenting with tachyarrhythmia. Is it type 1,2 or $4 \mathrm{c}$ myocardial infarction? Minerva Cardioangiol 2016;64:706-8.

6. Alexopoulos D. Acute myocardial infarction late following stent implantation: Incidence, mechanisms and clinical presentation. Int J Cardiol 2011;152:295-301.

7. Kotoku N, Higuma T, Ishibashi Y, et al. ST-segment elevation myocardial infarction caused by very late in-stent restenosis nine years after deployment. Coron Artery Dis 2020;31:742-3.

8. Thayssen P, Jensen LO, Lassen JF, et al. The risk and prognostic impact of definite stent thrombosis or instent restenosis after coronary stent implantation. EuroIntervention 2012;8:591-8.

9. Magalhaes MA, Minha S, Chen F, et al. Clinical presentation and outcomes of coronary in-stent restenosis across 3-stent generations. Circ Cardiovasc Interv 2014;7:768-76.

10. Roffi M, Patrono C, Collet JP, et al. 2015 ESC Guidelines for the management of acute coronary syndromes in patients presenting without persistent ST-segment elevation: Task Force for the Management of Acute Coronary Syndromes in Patients Presenting without Persistent ST-Segment Elevation of the European Society of Cardiology (ESC). Eur Heart J 2016;37:267-315.

11. Ponikowski P, Voors AA, Anker SD, et al. 2016 ESC Guidelines for the diagnosis and treatment of acute and chronic heart failure: The Task Force for the diagnosis and treatment of acute and chronic heart failure of the European Society of Cardiology (ESC)Developed with the special contribution of the Heart Failure Association (HFA) of the ESC. Eur Heart J 2016;37:2129-200.

12. Cutlip DE, Windecker S, Mehran R, et al. Clinical end points in coronary stent trials: a case for standardized definitions. Circulation 2007;115:2344-51.

13. Xhepa E, Bresha J, Joner M, et al. Clinical outcomes by optical characteristics of neointima and treatment modality in patients with coronary in-stent restenosis. EuroIntervention 2020. doi: 10.4244/EIJ-D-20-00662.

14. Sawlani NN, Bhatt DL. How to Decipher OCT After PCI. JACC Cardiovasc Imaging 2015;8:1306-8.

15. Bangalore S, Bhatt DL. Coronary intravascular ultrasound. Circulation 2013;127:e868-e874.

16. Theodoropoulos K, Mennuni MG, Dangas GD, et al. Resistant in-stent restenosis in the drug eluting stent era. Catheter Cardiovasc Interv 2016;88:777-85.

17. Kubo S, Kadota K, Otsuru S, et al. Optimal treatment of recurrent restenosis lesions after drug-eluting stent implantation for in-stent restenosis lesions. Eurointervention 2013:9:788-96.

18. Neumann FJ, Sousa-Uva M, Ahlsson A, et al. 2018 ESC/ EACTS Guidelines on myocardial revascularization. Eur Heart J 2019;40:87-165.

19. Giacoppo D, Alfonso F, Xu B, et al. Paclitaxel-coated balloon angioplasty vs. drug-eluting stenting for the treatment of coronary in-stent restenosis: a comprehensive, collaborative, individual patient data meta-analysis of 10 randomized clinical trials (DAEDALUS study). Eur Heart J 2020;41:3715-28.

20. Giacoppo D, Alfonso F, Xu B, et al. Drug-Coated Balloon Angioplasty Versus Drug-Eluting Stent Implantation in Patients With Coronary Stent Restenosis. J Am Coll Cardiol 2020;75:2664-78.

21. Belkacemi A, Agostoni P, Nathoe HM, et al. First results of the DEB-AMI (drug eluting balloon in acute ST-segment elevation myocardial infarction) trial: a multicenter randomized comparison of drug-eluting balloon plus baremetal stent versus bare-metal stent versus drug-eluting 
stent in primary percutaneous coronary intervention with 6-month angiographic, intravascular, functional, and clinical outcomes. J Am Coll Cardiol 2012;59:2327-37.

22. Fang CY, Fang HY, Chen CJ, et al. Comparison of clinical outcomes after drug-eluting balloon and drug-eluting

Cite this article as: Wang J, Gao H, Xiao J, Gao M, Liu Y, Gao J. The clinical features and prognosis of type 4C myocardial infarction in patients with non-ST-segment elevation myocardial infarction. Ann Transl Med 2021;9(14):1153. doi: 10.21037/atm-21-2587 stent use for in-stent restenosis related acute myocardial infarction: a retrospective study. PeerJ 2018;6:e4646.

(English Language Editor: J. Reynolds) 


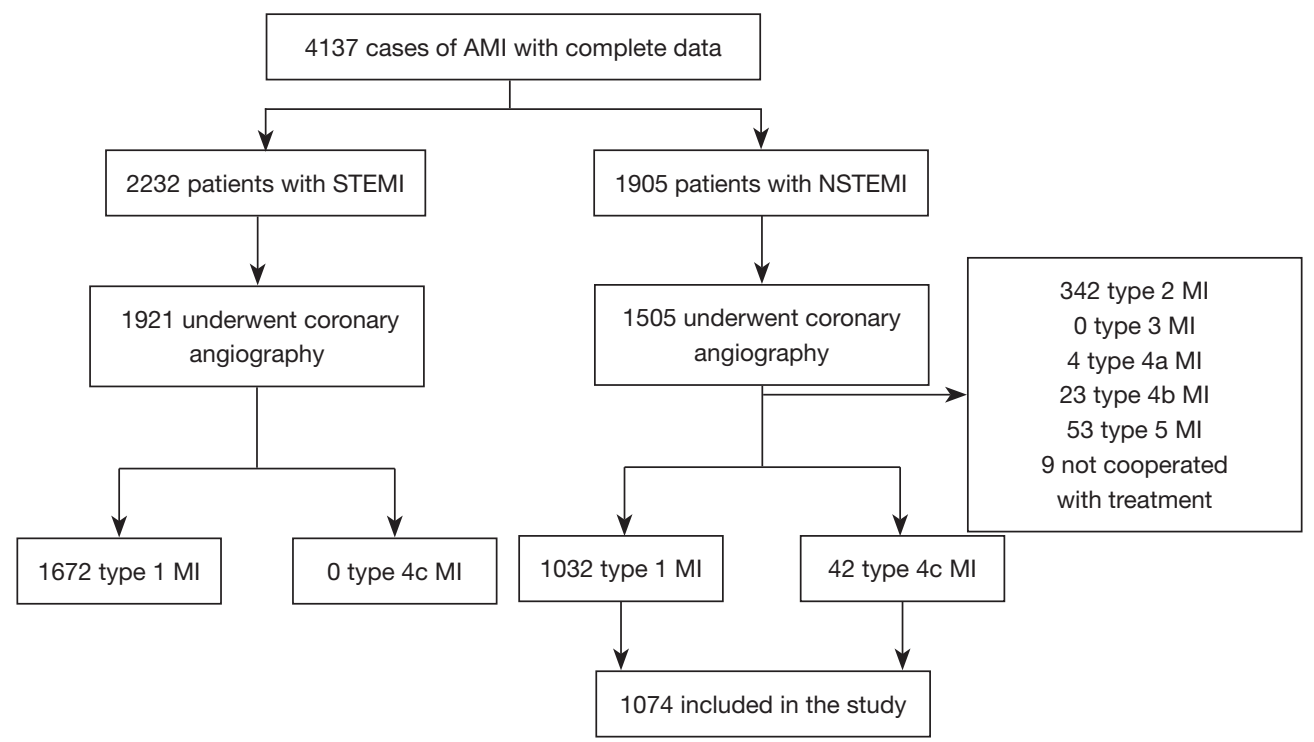

Figure S1 Screening flowchart. AMI, acute myocardial infarction; STEMI, ST-segment elevation myocardial infarction; NSTEMI, nonST-segment elevation myocardial infarction; MI, myocardial infarction. 


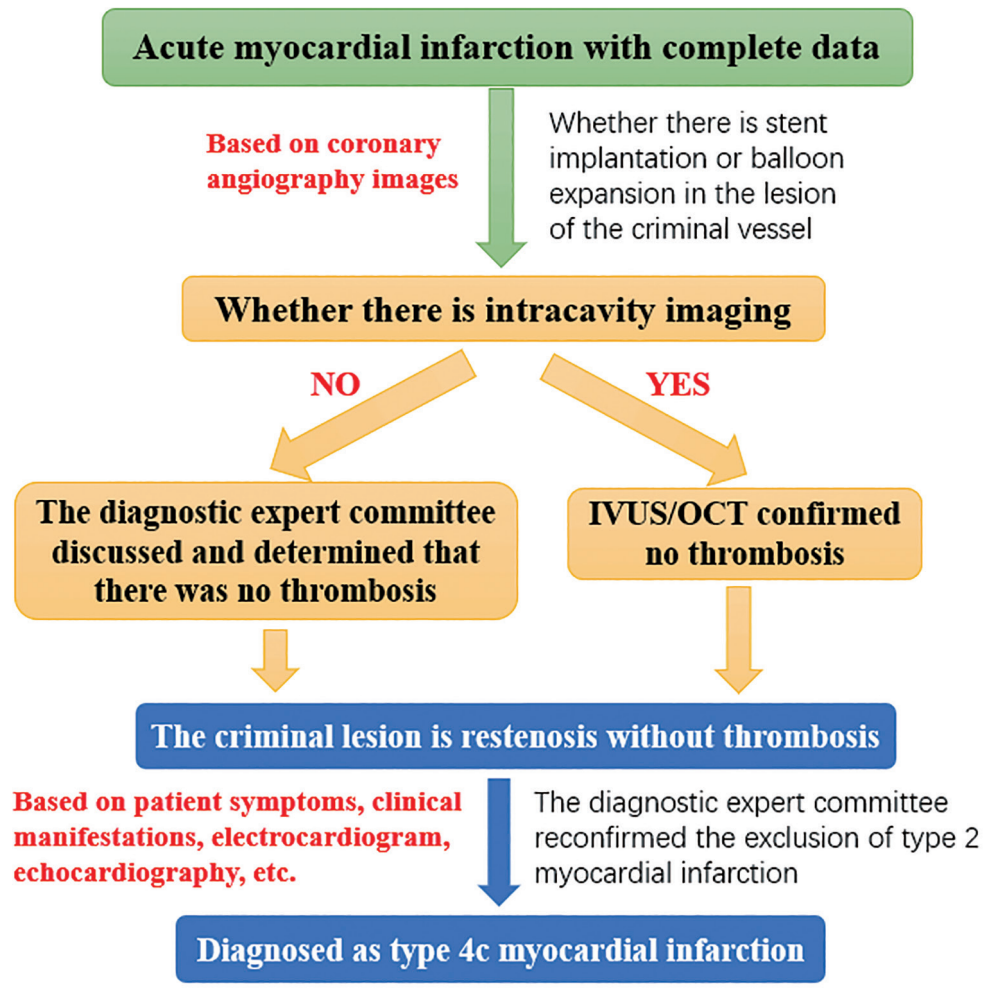

Figure S2 Flow chart for diagnosis of type 4c myocardial infarction. 

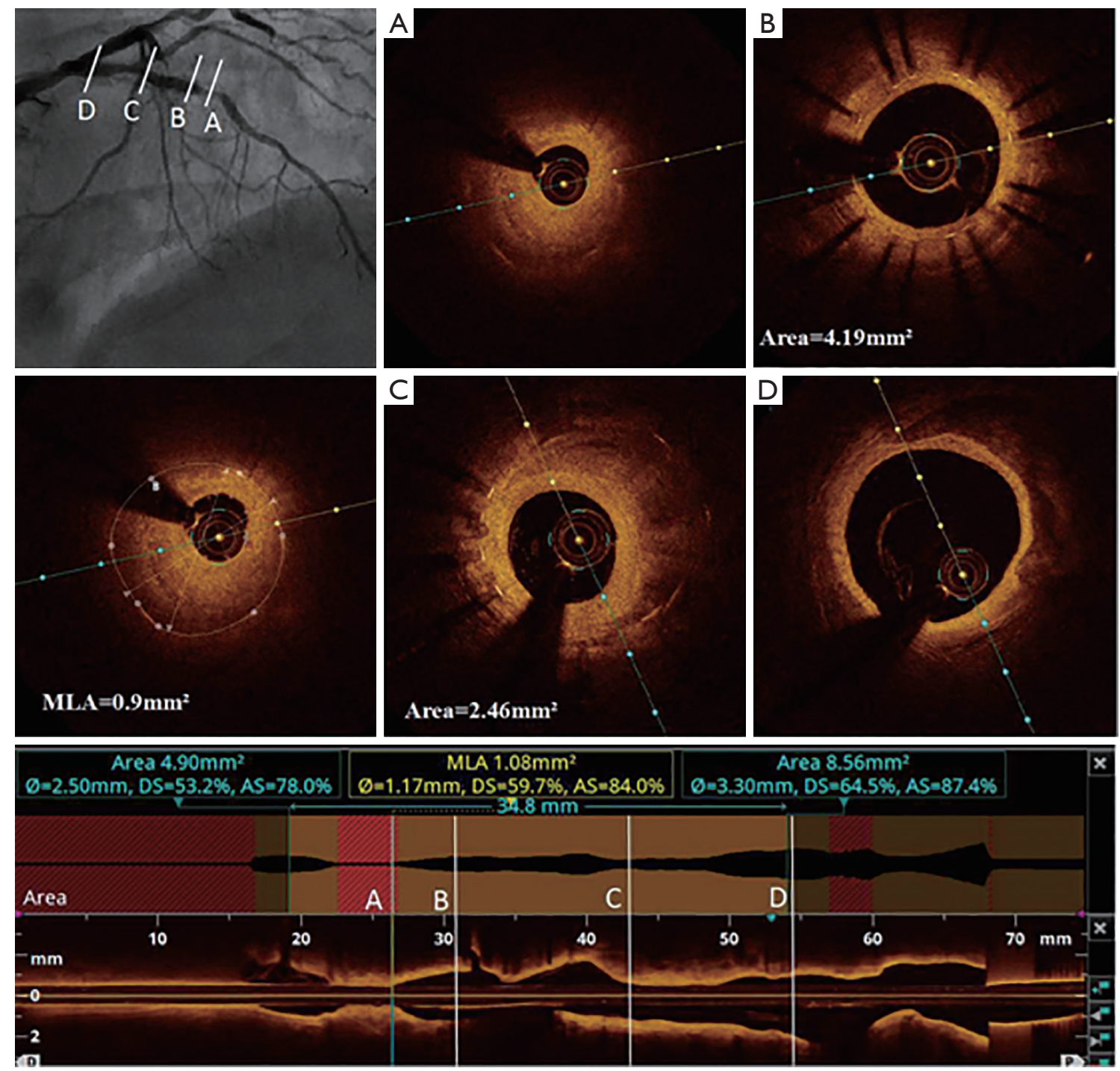

Figure S3 Case. 